\title{
Brokering trust: estimating the cost of physician-assisted death
}

\author{
Cheryl Mack, MD $\cdot$ Brendan Leier, PhD
}

Received: 17 November 2015/Accepted: 16 December 2015/Published online: 5 January 2016

(C) Canadian Anesthesiologists' Society 2016

In this issue of the Journal, Mottiar et al. ${ }^{1}$ provide an excellent summary of the current legal environment pertaining to physician-assisted death (PAD) and the possible implications for anesthesiologists in our country. What concerns us, from the perspective of front-line clinicians, is the lack of a real national dialogue on how this issue fundamentally challenges the goals of medicine and the relationship we have at the bedside with many of our most vulnerable patients. Physician-assisted death is not end-of-life care in the traditional sense of the term. This is evident in the practical implementation of physicianassisted suicide and euthanasia in other countries. ${ }^{2}$ Existential suffering and fears of losing control, being a burden, experiencing pain, and grappling with the unknown are real fears that cause tangible suffering, but we need to ask if there are better ways for physicians and communities to address this suffering.

Several questions need answering. Do we practice medicine in a system that provides sufficient access to social and mental health supports as well as the proper resources for palliative care? Do our patients and their

C. Mack, MD (ه)

Divisions of Adult Cardiothoracic and Pediatric Anesthesiology, Department of Anesthesiology and Pain Medicine, Division of Pediatric Palliative Care, and Department of Pediatrics, University of Alberta/Stollery Children's Hospitals and Mazankowski Heart Institute, John Dossetor Health Ethics Centre, 5-16 University Terrace, Edmonton, AB T6G 2T4, Canada

e-mail: cheryl.mack@shaw.ca

B. Leier, $\mathrm{PhD}$

John Dossetor Health Ethics Centre, Faculty of Medicine and Dentistry, University of Alberta, 5-16 University Terrace, Edmonton, AB T6G 2T4, Canada families feel isolated and abandoned as they try to deal with the challenges of a complex chronic condition? Do they feel unable to cope and become exhausted trying to meet the needs of their loved ones? Do our workers in the areas of social welfare, mental health, and palliative care consider they have the resources to meet the needs of our patients and their families? When patients do come to us with unremitting somatic and existential suffering, do we have the resources to help them? As physicians, perhaps we should imagine better ways to deal with these issues. Instead of advocating for physician-assisted suicide and euthanasia, we should be advocating for a solution to the root causes of these requests.

The Hippocratic tradition is not immutable, but any evolution of the goals or ethos of medicine that grounds the trust between physicians and their patients ought to be undertaken very seriously. Artificial timelines set by others are forcing us to truncate what ought to be a reflective dialogue among physicians and between physicians and their entire community of patients. The current legal and political climate of our nation and the interests of isolated advocacy groups cannot dictate a change that may have a serious impact on what it means to be a physician and provide care for our patients. This matter is not merely about one subset of patients; rather, we need to consider what impact a change in practice might have on all our patients. Our most vulnerable patients, in particular, need us to advocate for their lives in a very different way from what is being asked of us in PAD. We need to ensure that we advocate for a system that protects their interests and finds value in their lives.

While physicians did not advocate for or facilitate the recent Supreme Court decision, Carter v. Canada, ${ }^{3}$ physicians will bear a disproportionate responsibility and burden as a result. Setting predetermined, non-medical 
criteria for candidacy for assisted death places physicians in the uncomfortable position of administering a non-medical procedure or process to self-identified patients whose status was determined exclusively by subjective criteria (i.e., irremediable suffering). Our fundamental concern is that the proposed model of PAD does not require medical expertise; rather, it requires capital in the form of physician trust to assure both patients and society as a whole that the intentional ending of life is a legitimate medical procedure overseen with the same care, diligence, and oversight as any technological or procedural advancement. This, however, is not the case. Traditionally, in the rare circumstances in which individual physicians stray into practicing forms of "pseudomedicine", the greatest danger presented is a loss of trust in the profession as a whole. To illustrate this danger, we need only recall a recent and somber example of pseudo-medicine in the overextension of medical science into social and political eugenic policies. Eugenics and the selective nontreatment of infants born with trisomy 21 are the best, and perhaps most extreme, examples of situations where medical science was utilized as an appeal to authority to justify and rationalize bias. ${ }^{4}$ More recent instances might include physician product endorsement and physician conflict of interest in biased industry-sponsored research. ${ }^{5}$ With PAD, we are again being asked to endorse and sponsor a practice that relies neither on medical science nor on clinical judgment. This is precisely the danger of uncritical acceptance by provincial colleges and other medical associations implementing the practice of PAD. The potential loss of trust resulting from physicians being the de facto sponsors of assisted suicide and euthanasia is the loss of trust in doctors as doctors, a burden likely borne disproportionately by those who regularly care for the dying.

The law speaks rather narrowly of our professional ethics and obligations to our patients. The fact that an act is not illegal does not address the moral rightness or wrongness of an action in a profession that must engender the trust of its patients. Many actions are not illegal; all the same, is a physician obliged to engage in this conduct in the context of a physician-patient relationship wherein that action would be seen as a serious breach of trust? A physician found to breach that trust would be severely censured by our regulatory body (i.e., the provincial colleges). The reason for this is the unique aspect of our therapeutic relationship with our patients and its inherent imbalance of power. If physicians cross the line into intentional killing, even with consent, it might alter the trust our families require when we help them make critical decisions about possible withdrawal of life-sustaining therapy for a loved one in a very different situation. It raises the question whether our families would trust that we are honestly unable to provide the patient any benefit through further treatment or that we are instead simply trying to be expedient.
We do think that Mottiar et al. are correct to point out that anesthesiologists will be unable to avoid the issue. Indeed, some of us will almost certainly be asked to provide expert knowledge and, given our broad technical expertise and scope of practice, our direct involvement might be requested. As a result, we need to consider this matter carefully, use sound medical judgment, and seriously think through the highly contestable nature of what the courts (and perhaps our government) are asking us to undertake. In reflecting on the evolution of our professional obligations, we need to question whether physicians ought to be involved in assisted suicide and euthanasia in the first place and whether our involvement represents a morally acceptable change to our scope of practice. We suggest that the burden of proof lies with the Supreme Court to demonstrate the legitimacy of the nomination of physicians as the exclusive purveyors of a non-medical practice, particularly when the ethos of medicine has historically forbidden participation in this very act.

\section{L'enjeu de la confiance: que va nous coûter l'aide médicale à mourir?}

Dans ce numéro du Journal, Mottiar et coll. ${ }^{1}$ nous proposent un excellent résumé de l'environnement légal actuel qui entoure l'aide médicale à mourir (AMM) et ses implications possibles pour les anesthésiologistes canadiens. Ce qui nous préoccupe, en tant que cliniciens de première ligne, est l'absence totale d'un véritable dialogue national quant à la façon dont l'AMM remet fondamentalement en question les objectifs de la médecine et la relation que nous développons alors que nous sommes au chevet de bon nombre de nos patients les plus vulnérables. L'aide médicale à mourir n'équivaut pas à des soins de fin de vie au sens traditionnel du terme. Cette distinction est évidente dans l'application concrète de l'aide médicale au suicide et de l'euthanasie dans d'autres pays. ${ }^{2}$ Les souffrances existentielles et la peur de perdre le contrôle, de devenir un fardeau, d'avoir mal, et d'affronter l'inconnu sont des peurs véritables qui provoquent incontestablement des souffrances bien réelles. Toutefois, nous devons nous poser la question de savoir s'il existe de meilleures façons d'aborder et de traiter cette souffrance, en tant que médecins et en tant que collectivités.

Plusieurs questions ont besoin de réponses. Pratiquons-nous la médecine dans un système qui offre un accès suffisant aux programmes de soutien de la santé sociale et mentale, ainsi que des ressources adaptées de soins palliatifs? Nos patients et leurs familles se sentent-ils isolés et abandonnés lorsqu'ils 
tentent de faire face aux défis qu'engendre une maladie chronique complexe? Se sentent-ils incapables de tenir le coup? S'épuisent-ils en essayant de répondre aux besoins de leurs proches? Nos travailleurs dans les domaines de la prévention sociale, de la santé mentale et des soins palliatifs estiment-ils disposer des ressources nécessaires pour répondre aux besoins de nos patients et de leurs familles? Lorsque les patients nous approchent avec des souffrances somatiques et existentielles constantes, disposons-nous de ressources adaptées pour leur venir en aide? En tant que médecins, peut-être devrions-nous réfléchir à d'autres façons de répondre à ces problèmes. Plutôt que de préconiser l'aide médicale au suicide et l'euthanasie, nous devrions plaider en faveur d'une solution qui traite les causes à la base de ces demandes.

La tradition hippocratique n'est pas immuable, mais il est essentiel de réfléchir sérieusement à toute évolution des objectifs ou de l'ethos de la médecine qui sape la confiance entre le médecin et son patient. Des délais artificiels, décidés par autrui, nous forcent à écourter ce qui aurait dû être un dialogue empreint de réflexion parmi les médecins et entre les médecins et la communauté de patients dans son intégralité. Le climat politico-légal actuel de notre nation et les intérêts de groupes de pression isolés ne peuvent dicter un changement qui pourrait avoir un impact considérable sur la signification même de notre profession et notre rôle - soit de prodiguer des soins à nos patients. Cette question ne touche pas uniquement un petit sous-groupe de patients; au contraire, il nous faut réfléchir à l'impact qu'un tel changement dans notre pratique aura sur tous nos patients. Nos patients les plus vulnérables, en particulier, ont besoin de nous pour défendre leurs vies d'une manière fort différente de ce qu'on nous demande en AMM. Nous devons nous assurer que nous défendons un système qui protège leurs intérêts et accorde de la valeur à leurs vies.

Bien que les médecins n'aient ni encouragé, ni facilité la décision récente de la Cour suprême dans la cause Carter $c$. Canada, ${ }^{3}$ ce sont néanmoins eux qui porteront une responsabilité et un fardeau disproportionnés en conséquence de cette décision. La mise en place de critères prédéterminés et non médicaux pour être éligible à l'aide médicale à mourir place les médecins dans la position inconfortable de devoir administrer une procédure ou un processus non médical à des patients auto-identifiés et dont le statut a été déterminé en fonction de critères exclusivement subjectifs (une souffrance irrémédiable). Notre inquiétude principale est que le modèle proposé d'AMM ne nécessite aucune expertise médicale; en réalité, il s'appuie sur le capital que représente la confiance du public envers le médecin : ainsi, on compte rassurer tant les patients que la société en général que l'interruption intentionnelle d'une vie constitue une procédure médicale légitime supervisée avec autant de soin, de diligence et de supervision que tout autre progrès technologique ou procédural. Et pourtant, ce n'est pas le cas. Traditionnellement, dans les rares cas où des médecins isolés s'écartent de la norme acceptée et pratiquent des formes de « pseudo-médecine », le plus grand danger est une perte de confiance envers la profession dans son ensemble. Pour illustrer ce danger, souvenons-nous simplement d'un exemple récent et sombre de pseudo-médecine où la science médicale $\mathrm{a}$ outrepassé ses attributs pour s'immiscer dans des politiques d'eugénisme sociopolitique. L'eugénisme et le non-traitement sélectif de nourrissons nés avec une trisomie 21 sont les meilleurs exemples - voire les plus extrêmes - de situations où la science médicale a été utilisée pour appeler les autorités à justifier et rationnaliser un biais. ${ }^{4}$ Parmi les exemples plus récents, citons l'appui de médecins pour certains produits et les conflits d'intérêts de médecins impliqués dans des recherches biaisées financées par l'industrie. ${ }^{5}$ Dans le cas de l'AMM, on nous demande, une fois de plus, d'appuyer et de soutenir une pratique qui ne se fonde ni sur la science médicale, ni sur le jugement clinique. Voilà précisément le danger d'une approbation inconditionnelle par les collèges provinciaux et autres associations médicales mettant en œuvre la pratique de l'AMM. La potentielle perte de confiance émanant des médecins considérés, de facto, comme favorables à l'aide au suicide et à l'euthanasie, devient alors la perte de confiance en les médecins en tant que médecins; un fardeau disproportionnellement porté par ceux qui sont régulièrement en charge des soins de nos mourants.

La loi n'aborde que très peu les questions touchant à notre déontologie et à nos obligations professionnelles envers nos patients. La légalité ou l'illégalité d'un acte n'a aucun rapport avec sa justesse ou son absence de justesse morale, particulièrement dans notre profession, qui doit susciter la confiance de ses patients. De nombreux actes ne sont pas illégaux; nonobstant, un médecin est-il obligé de participer à un tel acte dans le contexte d'une relation de médecin à patient, c'est-à-dire dans un contexte où cet acte serait considéré comme un grave abus de confiance? Si l'on découvrait qu'un médecin avait abusé de cette confiance, il serait gravement censuré par son organisme de réglementation (c.-à-d. son collège provincial). Cette action punitive s'explique et se justifie par l'aspect unique de notre relation thérapeutique avec nos patients et du déséquilibre de pouvoir inhérent à cette relation. Si les médecins franchissaient le pas et tuaient intentionnellement, même si le patient est consentant, cela pourrait altérer la confiance dont nos familles ont besoin lorsque nous les aidons à prendre des décisions cruciales concernant l'interruption possible des traitements de maintien artificiel des fonctions vitales d'un être aimé dans une situation fondamentalement différente. Cela soulève la question suivante : les familles nous feront-elles confiance, pensant 
que nous ne pouvons pas, en toute honnêteté, procurer de bienfait supplémentaire à nos patients avec d'autres traitements, ou penseront-elles que nous essayons simplement de faire avancer les choses plus vite?

Nous sommes d'accord avec Mottiar et coll. lorsqu'ils soulignent que les anesthésiologistes ne pourront pas esquiver cette question. En effet, il est presque sûr que certains d'entre nous seront approchés afin d'offrir leurs connaissances d'experts et, étant donné notre vaste expertise technique et notre champ d'activité, notre implication directe pourrait être requise. C'est pourquoi nous devons évaluer cette question avec soin, faire preuve d'un jugement médical raisonné, et bien réfléchir à la nature très contestable de ce que les tribunaux (et notre gouvernement, dans une certaine mesure) nous demandent d'entreprendre. Dans le cadre de notre réflexion sur l'évolution de nos obligations professionnelles, nous devons nous poser la question de savoir si les médecins doivent, en premier lieu, s'impliquer dans l'aide au suicide et l'euthanasie, et si notre implication représente une modification acceptable, d'un point de vue moral, de notre champ d'activité. Nous suggérons que la charge de la preuve incombe à la Cour suprême, qui devra démontrer la légitimité de la nomination de médecins en tant que fournisseurs exclusifs d'une pratique non médicale, particulièrement lorsque l'ethos de la médecine interdit traditionnellement toute participation à cet acte.

Conflicts of interest None declared.

Conflit d'intérêt Aucun.

\section{References}

1. Mottiar M, Grant C, McVey MJ. Physician-assisted death and the anesthesiologist. Can J Anesth 2016; 63: this issue DOI: 10.1007/ s12630-015-0571-0.

2. Macleod $S$. Assisted dying in liberalised jurisdictions and the role of psychiatry: a clinician's view. Aust N Z J Psychiatry 2012; 46: 936-45.

3. Judgments of the Supreme Court of Canada. Carter v. Canada (Attorney General) 2015 SCC 5, 1 SCR 331. Available from URL: http://scc-csc.lexum.com/scc-csc/scc-csc/en/item/14637/index.do (accessed November 2015).

4. Mercurio MR. The Aftermath of Baby Doe and the Evolution of Newborn Intensive Care. Georgia State University Law Review 2008; 4: article 9. Available from URL: http://readingroom.law. gsu.edu/gsulr/vol25/iss4/9 (accessed November 2015).

5. CBS News. Dr. Oz-Endorsed Diet Pill Study was Bogus, Researchers Admit - October 2014. Available from URL: http://www.cbsnews. com/news/dr-oz-endorsed-green-coffee-bean-diet-study-retracted/ (accessed November 2015). 